\title{
Existence of positive periodic solutions for a generalized predator-prey model with diffusion feedback controls
}

\author{
Chaoxiong $\mathrm{Du}^{1 *}$ and Yusen $\mathrm{Wu}^{2}$
}

\section{"Correspondence:}

duchaoxiong123@163.com; ducx123@126.com

'Department of Mathematics, Shaoyang University, Shaoyang, Hunan 422000, P.R. China

Full list of author information is available at the end of the article

\begin{abstract}
By employing the continuation theorem of coincidence degree theory, we derive a sufficient condition for the existence and attractivity of at least a positive periodic solution of the generalized predator-prey model with exploited term.
\end{abstract}

MSC: $92 \mathrm{D} 25 ; 34 \mathrm{C} 25 ; 34 \mathrm{~K} 15$

Keywords: prey-predator model; diffusion; positive periodic solution; coincidence degree

\section{Introduction}

In recent years, the existence of positive periodic solutions of the prey-predator model has been widely studied [1-3]. The qualitative analysis of predator-prey systems is an interesting mathematical problem and has attracted a great attention of many mathematicians and biologists [4,5]. Recently, Xu and Chen [6] investigated the two-species ratio-dependent predator-prey different model with time delay. Since a realistic model requires the inclusion of the effect of changing environment, recently, Shihua and Feng [7] have considered the following model:

$$
\left\{\begin{aligned}
x_{1}^{\prime}(t)= & x_{1}(t)\left(a_{1}(t)-a_{11}(t) x_{1}(t)-\frac{a_{13}(t) x_{3}(t)}{m(t) x_{3}(t)+x_{1}(t)}\right) \\
& +D_{1}(t)\left(x_{2}(t)-x_{1}(t)\right)-h_{1}(t), \\
x_{2}^{\prime}(t)= & x_{2}(t)\left(a_{2}(t)-a_{22}(t) x_{2}(t)\right)+D_{2}(t)\left(x_{1}(t)-x_{2}(t)\right)-h_{2}(t), \\
x_{3}^{\prime}(t)= & x_{3}(t)\left(-a_{3}(t)-a_{4}(t) x_{3}(t)+\frac{a_{31}(t) x_{1}(t-\tau)}{m(t) x_{3}(t-\tau)+x_{1}(t-\tau)}\right)-h_{3}(t),
\end{aligned}\right.
$$

where $D_{i}(t)(i=1,2), a_{i}(t)(i=1,2,3), a_{11}(t), a_{13}(t), a_{22}(t), a_{31}(t)$ and $m(t)$ are strictly positive continuous $w$-periodic functions.

In the paper, we will study the following model:

$$
\left\{\begin{aligned}
x_{1}^{\prime}(t)= & x_{1}(t)\left(g_{1}\left(t, x_{1}(t)\right)-\frac{a_{13}(t) x_{3}(t)}{m(t) x_{3}(t)+x_{1}(t)}\right) \\
& +D_{1}(t)\left(x_{2}(t)-x_{1}(t)\right)-h_{1}(t), \\
x_{2}^{\prime}(t)= & x_{2}(t)\left(g_{2}\left(t, x_{2}(t)\right)+D_{2}(t)\left(x_{1}(t)-x_{2}(t)\right)-h_{2}(t),\right. \\
x_{3}^{\prime}(t)= & x_{3}(t)\left(-a_{3}(t)-a_{4}(t) x_{3}(t)+\frac{a_{31}(t) x_{1}(t-\tau)}{m(t) x_{3}(t-\tau)+x_{1}(t-\tau)}\right)-h_{3}(t),
\end{aligned}\right.
$$

(0) 2013 Du and Wu; licensee Springer. This is an Open Access article distributed under the terms of the Creative Commons Attribution License (http://creativecommons.org/licenses/by/2.0), which permits unrestricted use, distribution, and reproduction in any medium, provided the original work is properly cited. 
where $D_{i}(t)(i=1,2), a_{13}(t), a_{31}(t), m(t)$ are the same as in model (1.1). Some assumptions on the above functions on $g_{i}(t, x)(i=1,2)$ will be given in next section.

Our aim in this paper is to establish a sufficient condition for the existence and attractivity of at least a positive $w$-periodic solution of model (1.2).

\section{Main result}

To obtain the existence of positive periodic solutions of system (1.2), we summarize some concepts and results from [5] that will be basic for this section.

Let $X, Z$ be Banach spaces, let $L: \operatorname{Dom} L \subset X \rightarrow Y$ be a linear mapping, and let $N: X \rightarrow$ $Z$ be a continuous mapping. The mapping $L$ will be called a Fredholm mapping of index zero if $\operatorname{dim} \operatorname{Ker} L=\operatorname{codim} \operatorname{Im} L<+\infty$ and $\operatorname{Im} L$ is closed in $Z$. If $L$ is a Fredholm mapping of index zero, there exist continuous projectors $P: X \rightarrow Z$ and $Q: Z \rightarrow Z$ such that $\operatorname{Im} P=$ $\operatorname{Ker} L$ and $\operatorname{Im} L=\operatorname{Ker} Q=\operatorname{Im}(I-Q)$. It follows that $L / \operatorname{Dom} L \cap \operatorname{Ker} P:(I-P) X \rightarrow \operatorname{Im} L$ is invertible. We denote the inverse of that map by Kp. If $\Omega$ is an open-bounded subset of $X$, the mapping $N$ will be called $L$-compact on $\bar{\Omega}$ if $Q N(\bar{\Omega})$ is bounded and $\operatorname{Kp}(I-Q) N: \bar{\Omega} \rightarrow$ $X$ is compact. Since $\operatorname{Im} Q$ is isomorphic to $\operatorname{Ker} L$, there exists an isomorphism $J: \operatorname{Im} Q \rightarrow$ $\operatorname{Ker} L$.

In the proof of our existence theorem, we will use the continuation theorem of Gaines and Mawhin [8].

Lemma 2.1 [8] Let L be a Fredholm mapping of index zero and let $N$ be L-compact on $\bar{\Omega}$. Suppose the following:

(i) for each $\lambda \in(0,1)$, every solution $x$ of $L x=\lambda N x$ is such that $x \bar{\epsilon} \partial \Omega$;

(ii) $Q N x \neq 0$ for each $x \in \partial \Omega \cap \operatorname{Ker} L$;

(iii) $\operatorname{deg}\{J Q N, \Omega \cap \operatorname{Ker} L, 0\} \neq 0$.

The $L x=N x$ has at least one solution in $\operatorname{Dom} L \cap \bar{\Omega}$.

For convenience, we introduce the notations

$$
\bar{f}=\frac{1}{w} \int_{0}^{w} f(t) d t, \quad f^{L}=\min _{t \in[0, w]}|f(t)|, \quad f^{M}=\max _{t \in[0, w]}|f(t)|,
$$

where $f$ is a continuous $w$-periodic function.

Our main result on the global existence of a positive periodic solution of system (1.2) is stated in the following theorem.

Theorem 2.1 Assume that

$\left(\mathrm{H}_{1}\right)$ there exists a constant $A$ such that for $\forall x \in R, t \in R$, when $x \geq A$,

$$
g_{1}\left(t, e^{x}\right) \leq 0
$$

$\left(\mathrm{H}_{2}\right)$ there exists a constant $B$ such that for $\forall x \in R$, when $x \geq B$,

$$
g_{2}\left(t, e^{x}\right) \leq 0
$$

$\left(\mathrm{H}_{3}\right)$ there exists a constant $C(C<A)$ such that for $\forall x \in R, t \in R$, when $x \leq C$,

$$
e^{x} g_{1}\left(t, e^{x}\right) \geq\left(\frac{a_{13}}{m}\right)^{M} e^{x}+h_{1}^{M}
$$


$\left(\mathrm{H}_{4}\right)$ there exists a constant $D(D<B)$ such that for $\forall x \in R, t \in R$, when $x \leq D$,

$$
e^{x} g_{2}\left(t, e^{x}\right) \geq h_{2}^{M}
$$

$\left(\mathrm{H}_{5}\right) a_{31}^{M} e^{d_{1}}>h_{3}^{l} m^{l}$

Then system (1.1) has at least one positive w-periodic solution.

Proof Consider the system

$$
\left\{\begin{aligned}
u_{1}^{\prime}(t)= & g_{1}\left(t, e^{u_{1}(t)}\right)-\frac{a_{13}(t) e^{u_{3}(t)}}{m(t) e^{u_{3}(t)}+e^{u_{1}(t)}} \\
& +D_{1}(t)\left(e^{u_{2}(t)-u_{1}(t)}-1\right)-h_{1}(t) e^{-u_{1}(t)}, \\
u_{2}^{\prime}(t)= & g_{2}\left(t, e^{u_{2}(t)}\right)+D_{2}(t)\left(e^{u_{1}(t)-u_{2}(t)}-1\right)-h_{2}(t) e^{-u_{2}(t)}, \\
u_{3}^{\prime}(t)= & -a_{3}(t)-a_{4}(t) e^{u_{3}(t)}+\frac{a_{3}(t) e^{u_{1}(t-\tau)}}{m(t) e^{u_{3}(t)+e^{u_{1}(t-\tau)}}-h_{3}(t) e^{-u_{3}(t)}} .
\end{aligned}\right.
$$

Let $x_{i}(t)=e^{u_{i}(t)}, i=1,2,3$, then system (1.2) changes into system (2.1). Hence it is easy to see that system $(2.1)$ has a $w$-periodic solution $\left(u_{1}^{*}(t), u_{2}^{*}(t), u_{3}^{*}(t)\right)^{T}$, then $\left(e^{u_{1}^{*}(t)}, e^{u_{2}^{*}(t)}, e^{u_{3}^{*}(t)}\right)^{T}$ is a positive $w$-periodic solution of system (1.2). Therefore, for (1.2) to have at least one positive $w$-periodic solution, it is sufficient that (2.1) has at least one $w$-periodic solution. In order to apply Lemma 2.1 to system (2.1), we take

$$
X=Z=\left\{u(t)=\left(u_{1}(t), u_{2}(t), u_{3}(t)\right)^{T} \in C\left(R, R^{3}\right), u(t+w)=u(t)\right\}
$$

and

$$
\|u\|=\left\|\left(u_{1}(t), u_{2}(t), u_{3}(t)\right)^{T}\right\|=\sum_{i=1}^{3} \max _{t \in[0, w]}\left|u_{i}(t)\right|
$$

for any $u \in Z$ (or $Z$ ). Then $X$ and $Z$ are Banach spaces with the norm $\|\cdot\|$. Let

$$
\begin{aligned}
& N u=\left[\begin{array}{c}
g_{1}\left(t, e^{u_{1}(t)}\right)-\frac{a_{13}(t) e^{u_{3}(t)}}{m(t) e^{u_{3}(t)}+e^{u_{1}(t)}}+D_{1}(t)\left(e^{u_{2}(t)-u_{1}(t)}-1\right)-h_{1}(t) e^{-u_{1}(t)} \\
g_{2}\left(t, e^{u_{2}(t)}\right)+D_{2}(t)\left(e^{u_{1}(t)-u_{2}(t)}-1\right)-h_{2}(t) e^{-u_{2}(t)} \\
-a_{3}(t)-a_{4}(t) e^{u_{3}(t)}+\frac{a_{3}(t) e^{u_{1}(t-\tau)}}{m(t) e^{u_{3}(t)+e^{u_{1}(t-\tau)}}-h_{3}(t) e^{-u_{3}(t)}}
\end{array}\right], \quad u \in X, \\
& L u=u^{\prime}=\frac{d u(t)}{d t}, \quad p u=\frac{1}{w} \int_{0}^{w} u(t) d t, \quad u \in X ; \\
& Q z=\frac{1}{w} \int_{0}^{w} z(t) d t, \quad z \in Z .
\end{aligned}
$$

Then it follows that

$\operatorname{Ker} L=R^{3}, \quad \operatorname{Im} L=\left\{z \in Z: \int_{0}^{w} z(t) d t=0\right\}$ is closed in $Z$,

$\operatorname{dim} \operatorname{Ker} L=3=\operatorname{codim} \operatorname{Im} L$,

and $P, Q$ are continuous projectors such that

$\operatorname{Im} P=\operatorname{Ker} L, \quad \operatorname{Ker} Q=\operatorname{Im} L=\operatorname{Im}(I-Q)$. 
Therefore, $L$ is a Fredholm mapping of index zero. Furthermore, the generalized inverse (to $L$ ) $\operatorname{Kp}: \operatorname{Im} L \rightarrow \operatorname{Ker} P \cap \operatorname{Dom} L$ is given by

$$
\mathrm{Kp}(z)=\int_{0}^{t} z(s) d s-\frac{1}{w} \int_{0}^{w} \int_{0}^{t} z(s) d s d t
$$

Thus

$$
Q N u=\left[\begin{array}{l}
1 / w \int_{0}^{w} F_{1}(s) d s \\
1 / w \int_{0}^{w} F_{2}(s) d s \\
1 / w \int_{0}^{w} F_{3}(s) d s
\end{array}\right]
$$

and

$$
\mathrm{Kp}(I-Q) N u=\left[\begin{array}{l}
\int_{0}^{t} F_{1}(s) d s-1 / w \int_{0}^{w} \int_{0}^{t} F_{1}(s) d s d t+(1 / 2-t / w) \int_{0}^{w} F_{1}(s) d s \\
\int_{0}^{t} F_{2}(s) d s-1 / w \int_{0}^{w} \int_{0}^{t} F_{2}(s) d s d t+(1 / 2-t / w) \int_{0}^{w} F_{2}(s) d s \\
\int_{0}^{t} F_{3}(s) d s-1 / w \int_{0}^{w} \int_{0}^{t} F_{3}(s) d s d t+(1 / 2-t / w) \int_{0}^{w} F_{3}(s) d s
\end{array}\right],
$$

where

$$
\begin{aligned}
& F_{1}(s)=g_{1}\left(s, e^{u_{1}(s)}\right)-\frac{a_{13}(s) e^{u_{3}(s)}}{m(s) e^{u_{3}(s)}+e^{u_{1}(s)}}+D_{1}(s)\left(e^{u_{2}(s)-u_{1}(s)}-1\right)-h_{1}(s) e^{-u_{1}(s)}, \\
& F_{2}(s)=g_{2}\left(s, e^{u_{2}(s)}\right)+D_{2}(s)\left(e^{u_{1}(s)-u_{2}(s)}-1\right)-h_{2}(s) e^{-u_{2}(s)}
\end{aligned}
$$

and

$$
F_{3}(s)=-a_{3}(s)-a_{4}(s) e^{u_{3}(s)}+\frac{a_{3}(s) e^{u_{1}(s-\tau)}}{m(s) e^{u_{3}(s-\tau)}+e^{u_{1}(s-\tau)}}-h_{3}(s) e^{-u_{3}(s)} .
$$

Obviously, $Q N$ and $\mathrm{Kp}(I-Q) N$ are continuous. It is not difficult to show that $\mathrm{Kp}(I-$ Q) $N(\bar{\Omega})$ is compact for any open bounded $\Omega \subset X$ by using the Arzela-Ascoli theorem. Moreover, $Q N(\bar{\Omega})$ is clearly bounded. Thus, $N$ is $L$-compact on $\bar{\Omega}$ with any open bounded set $\Omega \subset X$.

Now we reach the point where we search for an appropriate open bounded subset $\Omega$ for the application of the continuation theorem (Lemma 2.1). Corresponding to the operator equation $L x=\lambda N x, \lambda \in(0,1)$, we have

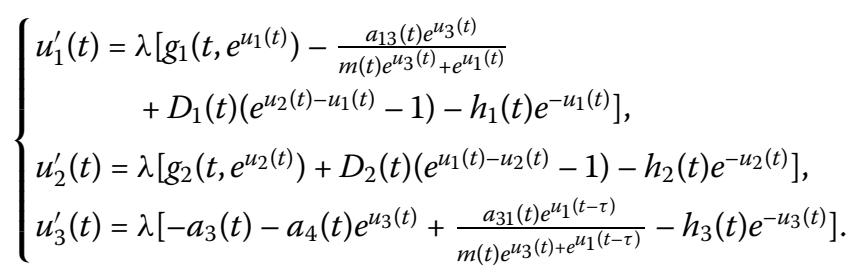

Assume that $u=u(t) \in X$ is a solution of system (2.2) for a certain $\lambda \in(0,1)$.

Because of $\left(u_{1}(t), u_{2}(t), u_{3}(t)\right)^{T} \in X$, there exist $\xi_{i}, \eta_{i} \in[0, w]$ such that

$$
u_{i}\left(\xi_{i}\right)=\max _{t \in[0, w]} u_{i}(t), \quad u_{i}\left(\eta_{i}\right)=\min _{t \in[0, w]} u_{i}(t), \quad i=1,2,3
$$


It is clear that

$$
u_{i}^{\prime}\left(\xi_{i}\right)=0, \quad u_{i}^{\prime}\left(\eta_{i}\right)=0, \quad i=1,2,3
$$

From this and system (2.2), we obtain

$$
\begin{aligned}
& g_{1}\left(\xi_{1}, e^{u_{1}\left(\xi_{1}\right)}\right)-\frac{a_{13}\left(\xi_{1}\right) e^{u_{3}\left(\xi_{1}\right)}}{m\left(\xi_{1}\right) e^{u_{3}\left(\xi_{1}\right)}+e^{u_{1}\left(\xi_{1}\right)}}+D_{1}\left(\xi_{1}\right)\left(e^{u_{2}\left(\xi_{1}\right)-u_{1}\left(\xi_{1}\right)}-1\right)-h_{1}\left(\xi_{1}\right) e^{-u_{1}\left(\xi_{1}\right)}=0, \\
& g_{2}\left(\xi_{2}, e^{u_{2}\left(\xi_{2}\right)}\right)+D_{2}\left(\xi_{2}\right)\left(e^{u_{1}\left(\xi_{2}\right)-u_{2}\left(\xi_{2}\right)}-1\right)-h_{2}\left(\xi_{2}\right) e^{-u_{2}\left(\xi_{2}\right)}=0, \\
& -a_{3}\left(\xi_{3}\right)-a_{4}\left(\xi_{3}\right) e^{u_{3}\left(\xi_{3}\right)}+\frac{a_{31}\left(\xi_{3}\right) e^{u_{1}\left(\xi_{3}-\tau\right)}}{m\left(\xi_{3}\right) e^{u_{3}\left(\xi_{3}-\tau\right)+e^{u_{1}\left(\xi_{3}-\tau\right)}}-h_{3}\left(\xi_{3}\right) e^{-u_{3}\left(\xi_{3}\right)}=0,} \\
& g_{1}\left(\eta_{1}, e^{u_{1}\left(\eta_{1}\right)}\right)-\frac{a_{13}\left(\eta_{1}\right) e^{u_{3}\left(\eta_{1}\right)}}{m\left(\eta_{1}\right) e^{u_{3}\left(\eta_{1}\right)}+e^{u_{1}\left(\eta_{1}\right)}}+D_{1}\left(\eta_{1}\right)\left(e^{u_{2}\left(\eta_{1}\right)-u_{1}\left(\eta_{1}\right)}-1\right) \\
& \quad-h_{1}\left(\eta_{1}\right) e^{-u_{1}\left(\eta_{1}\right)}=0, \\
& g_{2}\left(\eta_{2}, e^{u_{2}\left(\eta_{2}\right)}\right)+D_{2}\left(\eta_{2}\right)\left(e^{u_{1}\left(\eta_{2}\right)-u_{2}\left(\eta_{2}\right)}-1\right)-h_{2}\left(\eta_{2}\right) e^{-u_{2}\left(\eta_{2}\right)}=0, \\
& -a_{3}\left(\eta_{3}\right)-a_{4}\left(\eta_{3}\right) e^{u_{3}\left(\eta_{3}\right)}+\frac{a_{31}\left(\eta_{3}\right) e^{u_{1}\left(\eta_{3}-\tau\right)}}{m\left(\eta_{3}\right) e^{u_{3}\left(\eta_{3}-\tau\right)+e^{u_{1}\left(\eta_{3}-\tau\right)}}-h_{3}\left(\eta_{3}\right) e^{-u_{3}\left(\eta_{3}\right)}=0 .}
\end{aligned}
$$

There are two cases to consider for (2.3) and (2.4).

Case 1. Assume that $u_{1}\left(\xi_{1}\right) \geq u_{2}\left(\xi_{2}\right)$, then $u_{1}\left(\xi_{1}\right) \geq u_{2}\left(\xi_{1}\right)$.

From this and (2.3), we have

$$
g_{1}\left(\xi_{1}, e^{u_{1}\left(\xi_{1}\right)}\right)=\frac{a_{13}\left(\xi_{1}\right) e^{u_{3}\left(\xi_{3}\right)}}{m\left(\xi_{1}\right) e^{u_{3}\left(\xi_{1}\right)}+e^{u_{1}\left(\xi_{1}\right)}}-D_{1}\left(\xi_{1}\right)\left(e^{u_{2}\left(\xi_{1}\right)-u_{1}\left(\xi_{1}\right)}-1\right)+h_{1}\left(\xi_{1}\right) e^{-u_{1}\left(\xi_{1}\right)}>0,
$$

which, together with condition $\left(\mathrm{H}_{1}\right)$ in Theorem 2.1, gives

$$
u_{1}\left(\xi_{1}\right)<A
$$

Thus

$$
u_{2}\left(\xi_{2}\right) \leq u_{1}\left(\xi_{1}\right)<A
$$

Case 2. Assume that $u_{1}\left(\xi_{1}\right) \leq u_{2}\left(\xi_{2}\right)$, then $u_{1}\left(\xi_{2}\right)<u_{2}\left(\xi_{2}\right)$.

From this and (2.4), we have

$$
g_{2}\left(\xi_{2}, e^{u_{2}\left(\xi_{2}\right)}\right)=-D_{2}\left(\xi_{2}\right)\left(e^{u_{1}\left(\xi_{2}\right)-u_{2}\left(\xi_{2}\right)}-1\right)+h_{2}\left(\xi_{2}\right) e^{-u_{2}\left(\xi_{2}\right)}>0,
$$

which, together with condition $\left(\mathrm{H}_{2}\right)$ in Theorem 2.1, gives

$$
u_{2}\left(\xi_{2}\right)<B \text {. }
$$

Thus

$$
u_{1}\left(\xi_{1}\right) \leq u_{2}\left(\xi_{2}\right)<B
$$


From Case 1 and Case 2, we obtain

$$
\begin{aligned}
& u_{1}\left(\xi_{1}\right)<\max \{A, B\} \stackrel{\text { def }}{=} d_{1}, \\
& u_{2}\left(\xi_{2}\right)<\max \{A, B\}=d_{2} .
\end{aligned}
$$

From (2.5), we get

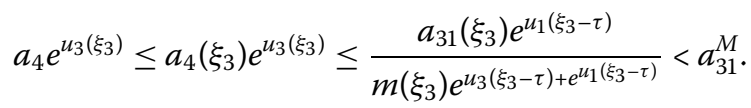

Thus

$$
u_{3}\left(\xi_{3}\right) \leq \ln \left(\frac{a_{31}^{M}}{a_{4}^{l}}\right) \stackrel{\text { def }}{=} d_{3}
$$

There are two cases to consider for (2.6) and (2.7).

Case 1 . Assume that $u_{1}\left(\eta_{1}\right) \leq u_{2}\left(\eta_{2}\right)$, then $u_{1}\left(\eta_{1}\right)<u_{2}\left(\eta_{1}\right)$. From this and (2.5), we have

$$
\begin{aligned}
g_{1}\left(\eta_{1}, e^{u_{1}\left(\eta_{1}\right)}\right) & =\frac{a_{13}\left(\eta_{1}\right) e^{u_{3}\left(\eta_{1}\right)}}{m\left(\eta_{1}\right) e^{u_{3}\left(\eta_{1}\right)}+e^{u_{1}\left(\eta_{1}\right)}}-D_{1}\left(\eta_{1}\right)\left(e^{u_{2}\left(\eta_{1}\right)-u_{1}\left(\eta_{1}\right)}-1\right)+h_{1}\left(\eta_{1}\right) e^{-u_{1}\left(\eta_{1}\right)} \\
& <\frac{a_{13}\left(\eta_{1}\right) e^{u_{3}\left(\eta_{1}\right)}}{m\left(\eta_{1}\right) e^{u_{3}\left(\eta_{1}\right)}+e^{u_{1}\left(\eta_{1}\right)}}+h_{1}\left(\eta_{1}\right) e^{-u_{1}\left(\eta_{1}\right)}<\left(\frac{a_{13}}{m}\right)^{M}+h_{1}^{M} e^{-u_{1}\left(\eta_{1}\right)}
\end{aligned}
$$

which, together with condition $\left(\mathrm{H}_{3}\right)$ in Theorem 2.1, gives

$$
u_{1}\left(\eta_{1}\right)>C
$$

Hence

$$
u_{2}\left(\eta_{2}\right)>u_{1}\left(\eta_{1}\right)>C
$$

Case 2. Assume that $u_{1}\left(\eta_{1}\right) \geq u_{2}\left(\eta_{2}\right)$, then $u_{1}\left(\eta_{2}\right) \geq u_{2}\left(\eta_{2}\right)$. From this and (2.7), we have

$$
g_{2}\left(\eta_{2}, e^{u_{2}\left(\eta_{2}\right)}\right)=-D_{2}\left(\eta_{2}\right)\left(e^{u_{1}\left(\eta_{2}\right)-u_{2}\left(\eta_{2}\right)}-1\right)+h_{2}\left(\eta_{2}\right) e^{-u_{2}\left(\eta_{2}\right)}<h_{2}^{M} e^{-u_{2}\left(\eta_{2}\right)}
$$

which, together with condition $\left(\mathrm{H}_{4}\right)$ in Theorem 2.1, gives

$$
u_{2}\left(\eta_{2}\right)>D
$$

Hence

$$
u_{1}\left(\eta_{1}\right)>u_{2}\left(\eta_{2}\right)>D \text {. }
$$

From Case 1 and Case 2, we have

$$
\begin{aligned}
& u_{1}\left(\eta_{1}\right)>\min \{C, D\} \stackrel{\text { def }}{=} \rho_{1}, \\
& u_{2}\left(\eta_{2}\right)>\min \{C, D\}=\rho_{1} .
\end{aligned}
$$


From Theorem $2.1\left(\mathrm{H}_{5}\right)$, we get

$$
u_{3}\left(\eta_{3}\right)>\ln \left(\frac{h_{3}^{l}}{a_{31}^{M}-a_{3}^{l}}\right) \text {. }
$$

From (2.11)-(2.22), we obtain, for $\forall t \in R$,

$$
\begin{aligned}
& \left|u_{1}(t)\right| \leq \max \left\{\left|d_{1}\right|,\left|\rho_{1}\right|\right\} \stackrel{\text { def }}{=} R_{1}, \\
& \left|u_{2}(t)\right| \leq \max \left\{\left|d_{1}\right|,\left|\rho_{1}\right|\right\} \stackrel{\text { def }}{=} R_{2}
\end{aligned}
$$

and

$$
\left|u_{3}(t)\right| \leq \max \left\{\left|a_{3}\right|,\left|\rho_{3}\right|\right\} \stackrel{\text { def }}{=} R_{3} .
$$

Clearly, $R_{i}(i=1,2,3)$ are independent of $\lambda$. Denote $M=\sum_{i=1}^{3} R_{i}+R_{0}$, here $R_{0}$ is taken sufficiently large such that each solution $\left(\alpha^{*}, \beta^{*}, \gamma^{*}\right)^{T}$ of the system

$$
\begin{aligned}
& g_{1}\left(t, e^{\alpha}\right)-\frac{\bar{a}_{13} e^{\gamma}}{m\left(t_{3}\right) e^{\gamma}+e^{\alpha}}+\bar{D}_{1}\left(e^{\beta-\alpha}-1\right)-\bar{h}_{1} e^{-\alpha}=0, \\
& g_{2}\left(t_{2}, e^{\beta}\right)+\bar{D}_{2}\left(e^{\alpha-\beta}-1\right)-\bar{h}_{2} e^{-\beta}=0 \\
& -\bar{a}_{3}-a_{4} e^{r}+\frac{\bar{a}_{31} e^{\alpha}}{m\left(t_{4}\right) e^{\gamma}+e^{\alpha}}-\bar{h}_{3} e^{-\gamma}=0
\end{aligned}
$$

satisfies $\left\|\left(\alpha^{*}, \beta^{*}, \gamma^{*}\right)^{T}\right\|=\left|\alpha^{*}\right|+\left|\beta^{*}\right|+\left|\gamma^{*}\right|<M$, provided that system (2.23) has a solution or a number of solutions, and that

$$
\max \left\{\left|d_{1}\right|,\left|\rho_{1}\right|\right\}+\max \left\{\left|d_{1}\right|,\left|\rho_{1}\right|\right\}+\max \left\{\left|d_{3}\right|,\left|\rho_{3}\right|\right\}<M
$$

where $t_{i} \in(0, w)$ will appear in $Q N u$ below.

Now we take $\Omega=\left\{u=\left(u_{1}(t), u_{2}(t), u_{3}(t)\right)^{T} \in X:\|u\|<M\right\}$. This satisfies condition (i) of Lemma 2.1. When $u \in \partial \Omega \cap \operatorname{Ker} L=\partial \Omega \cap R^{3}, u$ is a constant vector in $R^{3}$ with $\sum_{i=1}^{3}\left|u_{i}\right|=M$. If system (2.23) has one or more solutions, then

$$
Q N u=\left[\begin{array}{c}
g_{1}\left(t_{1}, e^{u_{1}}\right)-\frac{\bar{a}_{13} e^{u_{3}}}{m\left(t_{3}\right) e^{u_{3}+e^{u_{1}}}}+\bar{D}_{1}\left(e^{u_{2}-u_{1}}-1\right)-\bar{h}_{1} e^{-u_{1}} \\
g_{2}\left(t_{2}, e^{u_{2}}\right)+\bar{D}_{2}\left(e^{u_{1}-u_{2}}-1\right)-\bar{h}_{2} e^{-u_{2}} \\
-\bar{a}_{3}-\bar{a}_{4} e^{u_{3}}+\frac{\bar{a}_{13} e^{u_{1}}}{m\left(t_{4}\right) e^{u_{3}}+e^{u_{1}}}-\bar{h}_{3} e^{-u_{3}}
\end{array}\right] \neq(0,0,0)^{T},
$$

where $t_{i} \in(0, w)$ are one constant.

If system (2.23) does not have a solution, then naturally

$$
Q N u \neq(0,0,0)^{T}
$$

This shows that condition (ii) of Lemma 2.1 is satisfied finally. We will prove that condition (iii) of Lemma 2.1 is satisfied. We only prove that when $u \in \partial \Omega \cap \operatorname{Ker} L=\partial \Omega \cap R^{3}$, $\operatorname{deg}\left\{J Q N u, \partial \Omega \cap \operatorname{Ker} L,(0,0,0)^{T}\right\} \neq 0$. When $u \in \partial \Omega \cap \operatorname{Ker} L=\partial \Omega \cap R^{3}, u$ is a constant vector in $R^{3}$ with $\sum_{i=1}^{3}\left|u_{i}\right|=M$. Our proof will be broken into three steps as follows. 
Step 1. We prove

$$
\begin{aligned}
& \operatorname{deg}\left\{J Q N u, \Omega \cap \operatorname{Ker} L,(0,0,0)^{T}\right\} \\
& \quad=\operatorname{deg}\left\{\left(g\left(t_{1}, e^{u_{1}}\right), g\left(t_{2}, e^{u_{2}}\right),-\bar{a}_{3}-\bar{a}_{4} e^{u_{3}}+\frac{\bar{a}_{31} e^{u_{1}}}{m\left(t_{4}\right) e^{u_{3}}+e^{u_{1}}}-h_{3} e^{-u_{3}}\right)^{T},\right.
\end{aligned}
$$$$
\left.\Omega \cap \operatorname{Ker} L,(0,0,0)^{T}\right\} \text {. }
$$

To this end, we define the mapping $\phi_{1}: \operatorname{Dom} L \times[0,1] \rightarrow X$ by

$$
\begin{aligned}
\phi_{1}\left(u_{1}, u_{2}, u_{3}, \mu_{1}\right)= & {\left[\begin{array}{c}
g_{1}\left(t_{1}, e^{u_{1}}\right) \\
g_{2}\left(t_{2}, e^{u_{2}}\right) \\
-\bar{a}_{4} e^{u_{3}}+\frac{\bar{a}_{31} e^{u_{1}}}{m\left(t_{4}\right) e^{u_{3}}+e^{u_{1}}}
\end{array}\right] } \\
& +\mu_{1}\left[\begin{array}{c}
-\frac{\bar{a}_{13} u_{3}}{m\left(t_{3}\right) e^{u_{3}}+e^{u_{1}}}+\bar{D}_{1}\left(e^{u_{2}-u_{1}}-1\right)-\bar{h}_{1} e^{u_{1}} \\
\bar{D}_{2}\left(e^{u_{1}-u_{2}}-1\right)-\bar{h}_{2} e^{u_{2}} \\
-\bar{a}_{3}-\bar{h}_{3} e^{-u_{3}}
\end{array}\right],
\end{aligned}
$$

where $\mu_{1} \in[0,1]$ is a parameter, when $u=\left(u_{1}, u_{2}, u_{3}\right)^{T} \in \partial \Omega \cap \operatorname{Ker} L=\partial \Omega \cap R^{3}, u$ is a constant vector in $R^{3}$ with $\sum_{i=1}^{3}\left|u_{i}\right|=M$. We will show that when $u \in \partial \Omega \cap \operatorname{Ker} L$, $\phi_{1}\left(u_{1}, u_{2}, u_{3}, \mu_{1}\right) \neq 0$, if the conclusion is not true, i.e., the constant vector $u$ with $\sum_{i=1}^{3}\left|u_{i}\right|=$ $M$ satisfies $\phi_{1}\left(u_{1}, u_{2}, u_{3}, \mu_{1}\right)=0$, then from

$$
\begin{aligned}
& g_{1}\left(t_{1}, e^{u_{1}}\right)+\mu_{1}\left(\frac{-\bar{a}_{13} e^{u_{3}}}{m\left(t_{3}\right) e^{u_{3}}+e^{u_{1}}}+\bar{D}_{1}\left(e^{u_{2}-u_{1}}-1\right)\right)-\bar{h}_{1} e^{u_{1}}=0, \\
& g_{2}\left(t_{2}, e^{u_{2}}\right)+\mu_{1}\left(\bar{D}_{2}\left(e^{u_{1}-u_{2}}-1\right)-\bar{h}_{2} e^{u_{2}}\right)=0, \\
& -\bar{a}_{4} e^{u_{3}}+\frac{\bar{a}_{31} e^{u_{1}}}{m\left(t_{4}\right) e^{u_{3}}+e^{u_{1}}}-\mu_{1}\left(\bar{a}_{3}+\bar{h}_{3} e^{-u_{3}}\right)=0
\end{aligned}
$$

it follows the arguments of (2.11)-(2.22) that

$$
\left|u_{i}\right|<R_{i}, \quad i=1,2,3 .
$$

Thus

$$
\sum_{i=1}^{3}\left|u_{i}\right|<\sum_{i=1}^{3} R_{i}<M,
$$

which contradicts the fact that $\sum_{i=1}^{3}\left|u_{i}\right|=M$.

According to topological degree theory, we have

$$
\begin{aligned}
& \operatorname{deg}\left\{\left(J Q N, \Omega \cap \operatorname{Ker} L,(0,0,0)^{T}\right)\right\} \\
& \quad=\operatorname{deg}\left\{\phi_{1}\left(u_{1}, u_{2}, u_{3}, 1\right)^{T}, \Omega \cap \operatorname{Ker} L,(0,0,0)^{T}\right\} \\
& \quad=\operatorname{deg}\left\{\phi_{1}\left(u_{1}, u_{2}, u_{3}, 0\right)^{T}, \Omega \cap \operatorname{Ker} L,(0,0,0)^{T}\right\} \\
& \quad=\operatorname{deg}\left\{\left(g_{1}\left(t_{1}, e^{u_{1}}\right), g_{2}\left(t_{2}, e^{u_{2}}\right),-\bar{a}_{4} e^{u_{3}}+\frac{\bar{a}_{31} e^{u_{1}}}{m\left(t_{4}\right) e^{u_{3}}+e^{u_{1}}}\right)^{T}, \Omega \cap \operatorname{Ker} L,(0,0,0)^{T}\right\} .
\end{aligned}
$$


Step 2. We prove

$$
\begin{aligned}
& \operatorname{deg}\left\{\left(g_{1}\left(t_{1}, e^{u_{1}}\right), g_{2}\left(t_{2}, e^{u_{2}}\right),-\bar{a}_{4} e^{u_{3}}+\frac{\bar{a}_{31} e^{u_{1}}}{m\left(t_{4}\right) e^{u_{3}}+e^{u_{1}}}\right)^{T}, \Omega \cap \operatorname{Ker} L,(0,0,0)^{T}\right\} \\
& \quad=\operatorname{deg}\left\{\left(\bar{a}_{1}-\bar{a}_{11} e^{u_{1}}, g\left(t_{2}, e^{u_{2}}\right),-\bar{a}_{4} e^{u_{3}}+\frac{\bar{a}_{31} e^{u_{1}}}{m\left(t_{4}\right) e^{u_{3}}+e^{u_{1}}}\right)^{T}, \Omega \cap \operatorname{Ker} L,(0,0,0)^{T}\right\},
\end{aligned}
$$

where $\bar{a}_{1}, \bar{a}_{11}$ are two chosen positive constants such that

$$
C<\ln \frac{\bar{a}_{1}}{\bar{a}_{11}}<A
$$

To this end, we define the mapping $\phi_{2}: \operatorname{Dom} L \times[0,1] \rightarrow X$ by

$$
\begin{aligned}
& \phi_{2}\left(u_{1}, u_{2}, u_{3}, \mu_{2}\right)=\mu_{2}\left[\begin{array}{c}
\bar{a}_{1}-\bar{a}_{11} e^{u_{1}} \\
g_{2}\left(t_{2}, e^{u_{2}}\right) \\
-\bar{a}_{4} e^{u_{3}}+\frac{\bar{a}_{31} e^{u_{1}}}{m\left(t_{4}\right) e^{u_{3}}+e^{u_{1}}}
\end{array}\right] \\
& +\left(1-\mu_{2}\right)\left[\begin{array}{c}
g_{1}\left(t_{1}, e^{u_{1}}\right) \\
g_{2}\left(t_{2}, e^{u_{2}}\right) \\
-\bar{a}_{4} e^{u_{3}}+\frac{\bar{a}_{31} e^{u_{1}}}{m\left(t_{4}\right) e^{u_{3}}+e^{u_{1}}}
\end{array}\right] \\
& =\left[\begin{array}{c}
\mu_{2}\left(\bar{a}_{1}-\bar{a}_{11} e^{u_{1}}\right)+\left(1-\mu_{2}\right) g_{1}\left(t_{1}, e^{u_{1}}\right) \\
g_{2}\left(t_{2}, e^{u_{2}}\right) \\
-\bar{a}_{4} e^{u_{3}}+\frac{\bar{a}_{31} e^{u_{1}}}{m\left(t_{4}\right) e^{u_{3}}+e^{u_{1}}}
\end{array}\right],
\end{aligned}
$$

where $\mu_{2} \in[0,1]$ is a parameter. We will prove that when $u \in \partial \Omega \cap \operatorname{Ker} L, \phi_{2}\left(u_{1}, u_{2}, u_{3}, \mu_{2}\right) \neq$ $(0,0,0)^{T}$. When $u \in \partial \Omega \cap \operatorname{Ker} L=\partial \Omega \cap R^{3}, u$ is a constant vector in $R^{3}$ with $\sum_{i=1}^{3}\left|u_{i}\right|=M$. Now we consider two possible cases:
(i) $u_{1} \geq A$;
(ii) $u_{1}<A$.

(i) When $u_{1} \geq A$, from condition (iii) in Theorem 2.1, we have $g\left(t_{1}, e^{u_{1}}\right) \leq 0$. Moreover, $\bar{a}_{1}-\bar{a}_{11} e^{u_{1}} \leq \bar{a}_{1}-\bar{a}_{11} e^{A}<0$, thus $\mu_{2}\left(\bar{a}_{1}-\bar{a}_{11} e^{u_{1}}\right)+\left(1-\mu_{2}\right) g\left(t_{1}, e^{u_{1}}\right)<0$. Therefore, $\phi_{1}\left(u_{1}, u_{2}, u_{3}, \mu_{2}\right) \neq(0,0,0)^{T}$.

(ii) When $u_{1}<A$, if $u_{1} \leq C$, from condition $\left(\mathrm{H}_{3}\right)$ in Theorem 2.1, we have $g\left(t_{1}, e^{u_{1}}\right)>0$. However, $\bar{a}_{1}-\bar{a}_{11} e^{u_{1}} \geq \bar{a}_{1}-\bar{a}_{11} e^{C}>0$. Therefore, $\phi_{1}\left(u_{1}, u_{2}, u_{3}, \mu_{2}\right) \neq(0,0,0)^{T}$. If $u_{1}>C$, we also consider two possible cases: (a) $u_{2} \geq B$; (b) $u_{2}<B$. (a) When $u_{2} \geq B$, from condition $\left(\mathrm{H}_{2}\right)$ in Theorem 2.1, we have

$$
g_{2}\left(t_{2}, e^{u_{2}}\right)<0
$$

Therefore $\phi_{1}\left(u_{1}, u_{2}, u_{3}, \mu_{2}\right) \neq(0,0,0)^{T}$. (b) When $u_{2}<B$, if $u_{2} \leq D$, then from condition $\left(\mathrm{H}_{4}\right)$ in Theorem 2.1, we obtain $g_{2}\left(t_{2}, e^{u_{2}}\right)>0$. Consequently, $\phi_{2}\left(u_{1}, u_{2}, u_{3}, \mu_{2}\right) \neq(0,0,0)^{T}$. If $u_{2}>D$, we can claim when $u \in \partial \Omega \cap \operatorname{Ker} L=\partial \Omega \cap R^{3}, \phi_{2}\left(u_{1}, u_{2}, u_{3}, \mu_{2}\right) \neq(0,0,0)^{T}$. Otherwise, from

$$
-\bar{a}_{4} e^{u_{3}}+\frac{\bar{a}_{31} e^{u_{1}}}{m\left(t_{4}\right) e^{u_{3}}+e^{u_{1}}}=0
$$


we have

$$
e^{u_{3}}<\frac{\bar{a}_{31}}{\bar{a}_{4}}
$$

and

$$
e^{u_{3}}>\frac{-\bar{a}_{4} e^{\rho_{1}}+\sqrt{\left(-\bar{a}_{4} e^{\rho_{1}}\right)^{2}+4-\bar{a}_{4} m\left(t_{4}\right) \bar{a}_{31} e^{\rho_{1}}}}{2 \bar{a}_{4} m\left(t_{4}\right)}>0,
$$

i.e.,

$$
\begin{aligned}
& u_{3}<\ln \bar{a}_{31}-\ln \bar{a}_{4}, \\
& u_{3}>\ln \frac{-\bar{a}_{4} e^{\rho_{1}}+\sqrt{\left(-\bar{a}_{4} e^{\rho_{1}}\right)^{2}+4-\bar{a}_{4} m\left(t_{4}\right) \bar{a}_{31} e^{\rho_{1}}}}{2 \bar{a}_{4} m\left(t_{4}\right)} .
\end{aligned}
$$

Thus

$$
\begin{aligned}
& \left|u_{1}\right|<\max \left\{\left|d_{1}\right|,\left|\rho_{1}\right|\right\}, \\
& \left|u_{2}\right|<\max \left\{\left|d_{1}\right|,\left|\rho_{1}\right|\right\}
\end{aligned}
$$

and

$$
\left|u_{3}\right|<\max \left\{\left|d_{3}\right|,\left|\rho_{3}\right|\right\} .
$$

Therefore

$$
\begin{aligned}
\sum_{i=1}^{3}\left|u_{i}\right|< & \max \left\{\left|d_{1}\right|,\left|\rho_{1}\right|\right\}+\max \left\{\left|d_{1}\right|,\left|\rho_{1}\right|\right\} \\
& +\max \left\{\left|d_{3}\right|,\left|\rho_{3}\right|\right\}<M
\end{aligned}
$$

which contradicts the fact that $\sum_{i=1}^{3}\left|u_{i}\right|=M$. Based on the above discussion, for any $u \in$ $\partial \Omega \cap \operatorname{Ker} L$, we have $\phi_{2}\left(u_{1}, u_{2}, u_{3}, \mu_{2}\right) \neq(0,0,0)^{T}$. According to topological degree theory, we obtain

$$
\begin{aligned}
& \operatorname{deg}\left\{\left(g_{1}\left(t_{1}, e^{u_{1}}\right), g_{2}\left(t_{2}, e^{u_{2}}\right),-\bar{a}_{4} e^{u_{3}}+\frac{\bar{a}_{31} e^{u_{1}}}{m\left(t_{4}\right) e^{u_{3}}+e^{u_{1}}}\right)^{T}, \Omega \cap \operatorname{Ker} L,(0,0,0)^{T}\right\} \\
& =\operatorname{deg}\left\{\phi_{2}\left(u_{1}, u_{2}, u_{3}, 1\right)^{T}, \Omega \cap \operatorname{Ker} L,(0,0,0)^{T}\right\} \\
& =\operatorname{deg}\left\{\phi_{2}\left(u_{1}, u_{2}, u_{3}, 0\right)^{T}, \Omega \cap \operatorname{Ker} L,(0,0,0)^{T}\right\} \\
& =\operatorname{deg}\left\{\left(a_{1}-a_{11} e^{u_{1}}, g_{2}\left(t_{2}, e^{u_{2}}\right),-\bar{a}_{4} e^{u_{3}}+\frac{\bar{a}_{31} e^{u_{1}}}{m\left(t_{4}\right) e^{u_{3}}+e^{u_{1}}}\right)^{T}, \Omega \cap \operatorname{Ker} L,(0,0,0)^{T}\right\} .
\end{aligned}
$$

Step 3. We prove

$$
\begin{aligned}
& \operatorname{deg}\left\{\left(a_{1}-a_{11} e^{u_{1}}, g_{2}\left(t_{2}, e^{u_{2}}\right),-\bar{a}_{4} e^{u_{3}}+\frac{\bar{a}_{31} e^{u_{1}}}{m\left(t_{4}\right) e^{u_{3}}+e^{u_{1}}}\right)^{T}, \Omega \cap \operatorname{Ker} L,(0,0,0)^{T}\right\} \\
& \quad=\operatorname{deg}\left\{\left(a_{1}-a_{11} e^{u_{1}}, a_{2}-a_{22} e^{u_{2}},-\bar{a}_{4} e^{u_{3}}+\frac{\bar{a}_{31} e^{u_{1}}}{m\left(t_{4}\right) e^{u_{3}}+e^{u_{1}}}\right)^{T}, \Omega \cap \operatorname{Ker} L,(0,0,0)^{T}\right\} .
\end{aligned}
$$


To this end, we define the mapping $\phi_{3}: \operatorname{Dom} L \times[0,1] \rightarrow X$ by

$$
\begin{aligned}
\phi_{3}\left(u_{1}, u_{2}, u_{3}, \mu_{3}\right)= & \mu_{3}\left[\begin{array}{c}
a_{1}-a_{11} e^{u_{1}} \\
a_{2}-a_{22} e^{u_{2}} \\
-\bar{a}_{4} e^{u_{3}}+\frac{\bar{a}_{31} e^{u_{1}}}{m\left(t_{4}\right) e^{u_{3}}+e^{u_{1}}}
\end{array}\right] \\
& +\left(1-\mu_{3}\right)\left[\begin{array}{c}
a_{1}-a_{11} e^{u_{1}} \\
g_{2}\left(t_{2}, e^{u_{2}}\right) \\
-\bar{a}_{4} e^{u_{3}}+\frac{\bar{a}_{31} e^{u_{1}}}{m\left(t_{4}\right) e^{u_{3}}+e^{u_{1}}}
\end{array}\right] \\
= & {\left[\begin{array}{c}
a_{1}-a_{11} e^{u_{1}} \\
\mu_{3}\left(\bar{a}_{2}-\bar{a}_{22} e^{u_{2}}\right)+\left(1-\mu_{3}\right) g\left(t_{2}, e^{u_{2}}\right) \\
-\bar{a}_{4} e^{u_{3}}+\frac{\bar{a}_{31} e_{1}}{m\left(t_{4}\right) e^{u_{3}}+e^{u_{1}}}
\end{array}\right], }
\end{aligned}
$$

where $\mu_{3} \in[0,1]$ is a parameter and $a_{2}, a_{22}$ are two chosen positive constants such that $D<$ $\ln \frac{a_{2}}{a_{22}}<B$. We will prove that when $u \in \partial \Omega \cap \operatorname{Ker} L, \phi_{3}\left(u_{1}, u_{2}, u_{3}, \mu_{2}\right) \neq(0,0,0)^{T}$. If it is not true, then the constant vector $u$ satisfies $\phi_{3}\left(u_{1}, u_{2}, u_{3}, \mu_{2}\right) \neq(0,0,0)^{T}$ with $\sum_{i=1}^{3}\left|u_{i}\right|=M$. Thus we have

$$
\left\{\begin{array}{l}
a_{1}-a_{11} e^{u_{1}}=0, \\
\mu_{3}\left(a_{2}-a_{22} e^{u_{2}}\right)+\left(1-\mu_{3}\right) g_{2}\left(t_{2}, e^{u_{2}}\right)=0 \\
-\bar{a}_{4} e^{u_{3}}+\frac{\bar{a}_{31} e^{u_{1}}}{m\left(t_{4}\right) e^{u_{3}}+e^{u_{1}}}=0 .
\end{array}\right.
$$

(2.24) implies

$$
C<u_{1}=\ln \frac{a_{1}}{a_{11}}<A
$$

We claim that $u_{2}<B$; otherwise, if $u_{2} \geq B$, then from condition $\left(\mathrm{H}_{2}\right)$ in Theorem 2.1, we have

$$
\left(1-u_{3}\right) g_{2}\left(t_{2}, e^{u_{2}}\right)<0
$$

Consequently,

$$
\mu_{3}\left(a_{2}-a_{22} e^{u_{2}}\right)+\left(1-\mu_{3}\right) g_{2}\left(t_{2}, e^{u_{2}}\right)<0
$$

which contradicts (2.23). We also claim that $u_{2}>D$. If $u_{2} \leq D$, then $g_{2}\left(t_{2}, e^{u_{2}}\right)>0$. However, $a_{2}-a_{22} e^{u_{2}}>a_{2}-a_{22} e^{D}>0$.

Thus

$$
u_{3}\left(a_{2}-a_{22} e^{u_{2}}\right)+\left(1-\mu_{3}\right) g_{2}\left(t_{2}, e^{u_{2}}\right)>0
$$

which contradicts (2.24). (2.26) gives

$$
-\bar{a}_{4} e^{u_{3}}+\frac{\bar{a}_{31} e^{u_{1}}}{m\left(t_{4}\right) e^{u_{3}}+e^{u_{1}}}=0,
$$


that is,

$$
\begin{aligned}
& u_{3}<\ln \bar{a}_{31}-\ln \bar{a}_{4}, \\
& u_{3}>\ln \frac{-\bar{a}_{4} e^{\rho_{1}}+\sqrt{\left(-\bar{a}_{4} e^{\rho_{1}}\right)^{2}+4-\bar{a}_{4} m\left(t_{4}\right) \bar{a}_{31} e^{\rho_{1}}}}{2 \bar{a}_{4} m\left(t_{4}\right)} .
\end{aligned}
$$

Thus

$$
\begin{aligned}
& \left|u_{1}\right|<\max \left\{\left|d_{1}\right|,\left|\rho_{1}\right|\right\}, \\
& \left|u_{2}\right|<\max \left\{\left|d_{1}\right|,\left|\rho_{1}\right|\right\}
\end{aligned}
$$

and

$$
\left|u_{3}\right|<\max \left\{\left|d_{3}\right|,\left|\rho_{3}\right|\right\} \text {. }
$$

Therefore

$$
\begin{gathered}
\sum_{i=1}^{3}\left|u_{i}\right|<\max \left\{\left|d_{1}\right|,\left|\rho_{1}\right|\right\}+\max \left\{\left|d_{1}\right|,\left|\rho_{1}\right|\right\} \\
+\max \left\{\left|d_{3}\right|,\left|\rho_{3}\right|\right\}<M
\end{gathered}
$$

which leads to a contradiction. Therefore, by means of topological degree theory, we have

$$
\begin{aligned}
& \operatorname{deg}\left\{\left(a_{1}-a_{11} e^{u_{1}}, g_{2}\left(t_{2}, e^{u_{2}}\right),-\bar{a}_{4} e^{u_{3}}+\frac{\bar{a}_{31} e^{u_{1}}}{m\left(t_{4}\right) e^{u_{3}}+e^{u_{1}}}\right), \Omega \cap \operatorname{Ker} L,(0,0,0)^{T}\right\} \\
& =\operatorname{deg}\left\{\phi_{3}\left(u_{1}, u_{2}, u_{3}, 1\right)^{T}, \Omega \cap \operatorname{Ker} L,(0,0,0)^{T}\right\} \\
& =\operatorname{deg}\left\{\phi_{3}\left(u_{1}, u_{2}, u_{3}, 0\right)^{T}, \Omega \cap \operatorname{Ker} L,(0,0,0)^{T}\right\} \\
& =\operatorname{deg}\left\{\left(a_{1}-a_{11} e^{u_{1}}, a_{2}-a_{22} e^{u_{2}},-\bar{a}_{4} e^{u_{3}}+\frac{\bar{a}_{31} e^{u_{1}}}{m\left(t_{4}\right) e^{u_{3}}+e^{u_{1}}}\right), \Omega \cap \operatorname{Ker} L,(0,0,0)^{T}\right\} .
\end{aligned}
$$

From the proof of the three steps above, we obtain

$$
\begin{aligned}
& \operatorname{deg}\left\{J Q N u, \Omega \cap \operatorname{Ker} L,(0,0,0)^{T}\right\} \\
& \quad=\operatorname{deg}\left\{\left(a_{1}-a_{11} e^{u_{1}}, a_{2}-a_{22} e^{u_{2}},-\bar{a}_{4} e^{u_{3}}+\frac{\bar{a}_{31} e^{u_{1}}}{m\left(t_{4}\right) e^{u_{3}}+e^{u_{1}}}\right), \Omega \cap \operatorname{Ker} L,(0,0,0)^{T}\right\} .
\end{aligned}
$$

Because of condition $\left(\mathrm{H}_{5}\right)$ in Theorem 2.1, the system of algebraic equations

$$
\left\{\begin{array}{l}
a_{1}-a_{11} x=0, \\
a_{2}-a_{22} y=0, \\
-\bar{a}_{4} I+\frac{\bar{a}_{31} x}{m\left(t_{4}\right) z+x}=0
\end{array}\right.
$$

has a unique solution $\left(x^{*}, y^{*}, z^{*}\right)^{T}$ which satisfies

$$
x^{*}=\frac{a_{1}}{a_{11}}>0, \quad y^{*}=\frac{a_{2}}{a_{22}}>0, \quad z^{*}=\frac{a_{4} x^{*}+\sqrt{\left(a_{4} x^{*}\right)^{2}+4 a_{4} m\left(t_{4}\right) \bar{a}_{31} x^{*}}}{2 \bar{a}_{4} m\left(t_{4}\right)}>0 .
$$


Thus

$$
\begin{aligned}
& \operatorname{deg}\left\{\left(a_{1}-a_{11} e^{u_{1}}, a_{2}-a_{22} e^{u_{2}},-\bar{a}_{4} e^{u_{3}}+\frac{\bar{a}_{31} e^{u_{1}}}{m\left(t_{4}\right) e^{u_{3}}+e^{u_{1}}}\right), \Omega \cap \operatorname{Ker} L,(0,0,0)^{T}\right\} \\
& =\operatorname{sign}\left|\begin{array}{ccc}
-a_{11} x^{*} & 0 & 0 \\
0 & -a_{22} y^{*} & 0 \\
\ldots & 0 & -\bar{a}_{4} z^{*}-\frac{\bar{a}_{31} m\left(t_{4}\right) x^{*} z^{*}}{\left[m\left(t_{4}\right) z^{*}+x^{*}\right]^{2}}
\end{array}\right|=-1 .
\end{aligned}
$$

Therefore, from (2.20), we have

$$
\operatorname{deg}\left\{J Q N u, \Omega \cap \operatorname{Ker} L,(0,0,0)^{T}\right\}=-1 .
$$

This completes the proof of Theorem 2.1.

\section{An example}

Consider the system

$$
\left\{\begin{array}{l}
x_{1}^{\prime}=x_{1}(t)\left(a_{1}(t)-a_{11}(t) x_{1}(t)-\frac{a_{13}(t) x_{3}(t)}{m(t) x_{3}(t)+x_{1}(t)}\right)+D_{1}(t)\left(x_{2}(t)-x_{1}(t)\right) \\
x_{2}^{\prime}=x_{2}(t)\left(a_{2}(t)-a_{22}(t) x_{2}(t)\right)+D_{2}(t)\left(x_{1}(t)-x_{2}(t)\right) \\
x_{3}^{\prime}=x_{3}(t)\left(-a_{3}(t)+\frac{a_{31}(t) x_{1}(t-T)}{m(t) x_{3}(t-T)+x_{1}(t-T)}\right)
\end{array}\right.
$$

where $\tau>0$ is a positive constant, all the parameters are positive continuous $w$-periodic functions with periodic $w>0$.

In Theorem 2.1, $g_{1}\left(t, e^{x}\right)=a_{1}(t)-a_{11}(t) e^{x}, g_{2}\left(t, e^{x}\right)=a_{2}(t)-a_{22}(t) e^{x}$. It is easily shown that if $x \geq \ln \left(\frac{a_{1}^{m}}{a_{11}^{l}}\right)=A$, then $g_{1}\left(t, e^{x}\right) \leq 0$ and if $x \geq \ln \left(\frac{a_{2}^{M}}{a_{22}^{l}}\right)=B$, then $g_{2}\left(t, e^{x}\right) \leq 0$. We also can show if

$$
\begin{aligned}
& x \leq \ln \frac{a_{1}^{M}-\left(\frac{a_{13}}{m}\right)^{M}}{a_{11}^{l}}=C, \\
& g_{1}\left(t, e^{x}\right) \geq\left(\frac{a_{11}}{m}\right)^{M}
\end{aligned}
$$

and if $x \leq \ln \frac{a_{2}^{M}}{a_{22}^{l}}=D$, then $g_{2}\left(t, e^{x}\right)>0$.

$\left(\mathrm{H}_{1}\right) a_{1}^{m}>\left(\frac{a_{13}}{m}\right)^{M}$

$\left(\mathrm{H}_{2}\right) \bar{a}_{31}>\bar{a}_{3}$.

By Theorem 2.1, we have the following theorem.

Theorem 3.1 If $\left(\mathrm{H}_{1}\right)$ and $\left(\mathrm{H}_{2}\right)$ hold, the system (3.1) has at least one positive w-periodic solution. Consider the system

$$
\left\{\begin{array}{l}
x_{1}^{\prime}=x_{1}(t)\left(a_{1}(t)-a_{11}(t) x_{1}(t)-\frac{a_{13}(t) x_{1}(t) x_{3}(t)}{m(t) x_{3}^{2}(t)+x_{1}^{2}(t)}\right)+D_{1}(t)\left(x_{2}(t)-x_{1}(t)\right), \\
x_{2}^{\prime}=x_{2}(t)\left(a_{2}(t)-a_{22}(t) x_{2}(t)\right)+D_{2}(t)\left(x_{1}(t)-x_{2}(t)\right) \\
x_{3}^{\prime}=x_{3}(t)\left(-a_{3}(t)+\frac{a_{31}(t) x_{1}^{2}(t-\tau)}{m(t) x_{3}^{2}(t-\tau)+x_{1}^{2}(t-\tau)}\right)
\end{array}\right.
$$


where $z>0$ is a positive constant, all the parameters are positive continuous $w$-periodic functions with periodic $w>0$.

In Theorem 2.1, $g_{1}\left(t, e^{x}\right)=a_{1}(t)-a_{11}(t) e^{x}, g_{2}\left(t, e^{x}\right)=a_{2}(t)-a_{22}(t) e^{x}$. It is easily shown that if $x \geq \ln \frac{a_{1}^{M}}{a_{11}^{l}}=A$, then $g_{1}\left(t, e^{x}\right) \leq 0$ and if $x \geq \ln \frac{a_{2}^{M}}{a_{22}^{l}}=B$, then $g_{2}\left(t, e^{x}\right) \leq 0$. We also can show if $x \leq \ln \frac{a_{1}^{M}-\left(\frac{a_{13}}{2 \sqrt{m}}\right)^{M}}{a_{11}^{l}}=C, g_{1}\left(t, e^{x}\right) \geq\left(\frac{a_{13}}{2 \sqrt{m}}\right)^{m}$ and if $x \leq \ln \frac{a_{2}^{M}}{a_{22}^{l}}=D$, then $g_{2}\left(t, e^{x}\right) \geq 0$.

$\left(\mathrm{H}_{1}^{\prime}\right) \quad a_{1}^{M}>\left(\frac{a_{13}}{2 \sqrt{m}}\right)^{M}$,

$\left(\mathrm{H}_{2}^{\prime}\right) \bar{a}_{31}>\bar{a}_{3}$.

By Theorem 2.1, we have the following theorem.

Theorem 3.2 If $\left(\mathrm{H}_{1}^{\prime}\right)$ and $\left(\mathrm{H}_{2}^{\prime}\right)$ hold, system (3.2) has at least one positive w-periodic solution.

\section{Competing interests}

The authors declare that they have no competing interests.

Authors' contributions

Each of the authors, CD and YW, contributed to each part of this study equally and read and approved the final version of the manuscript.

\section{Author details}

'Department of Mathematics, Shaoyang University, Shaoyang, Hunan 422000, P.R. China. ${ }^{2}$ School of Mathematics and Statistics, Henan University of Science and Technology, Luoyang, Henan 471003, P.R. China.

\section{Acknowledgements}

This research is partially supported by the National Natural Science Foundation of China (11071222, 11101126), the Hunan provincial Natural Science Foundation of China (12JJ3008) and the Research Fund of Hunan provincial Education Department (11B113).

Received: 27 November 2012 Accepted: 15 February 2013 Published: 8 March 2013

\section{References}

1. Li, YK: Positive periodic solution of neutral predator-prey system. Appl. Math. Mech. 20(5), 545-550 (1999) (in Chinese)

2. Zhang, ZQ, Wang, ZC: The existence of a periodic solution for a generalized prey-predator system with delay. Math. Proc. Camb. Philos. Soc. 137, 475-487 (2004)

3. Zhang, ZQ, Zeng, XW: Periodic solution of a nonautonomous stage-structured single species model with diffusion. Q. Appl. Math. 63(2), 277-289 (2005)

4. Jose, F, Santiago, V: An approximation for prey-predator models with time delay. Physica D 110(3-4), 313-322 (1997)

5. Xiao, YN, Chen, LS: Modeling and analysis of predator-prey model with disease in the prey. Math. Biosci. 171(1), 59-82 (2001)

6. Xu, R, Chen, LS: Persistence and stability for two-species ratio-dependent predator-prey system with time delay in a two-patch environment. Comput. Math. Appl. 40, 577-588 (2000)

7. Shihua, C, Feng, W: Positive periodic solution of two-species ratio-dependent predator-prey system with time delay in two-patch environment. Appl. Math. Comput. 150, 737-748 (2004)

8. Gaines, RE, Mawhin, JL: Coincidence Degree and Non-Linear Differential Equations. Springer, Berlin (1977) 